\title{
YSOs in Taurus-Auriga-Perseus and Orion
}

\author{
Sarolta Zahorecz ${ }^{1}$, L. Viktor Tóth ${ }^{1}$, Gábor Marton ${ }^{2}$, \\ Toshikazu Onishi ${ }^{3}$, Lajos G. Balázs ${ }^{2}$, Orsolya Fehér ${ }^{1}$, \\ Akiko Kawamura $^{4}$, Yoshimi Kitamura ${ }^{5}$, Mónika Lisztes ${ }^{1}$, \\ Atsushi Nishimura ${ }^{3}$, László Pásztor ${ }^{6}$, Sándor Pintér ${ }^{1}$, István Rácz ${ }^{1}$, \\ Motohide Tamura ${ }^{4}$, Rogel M.D. Sese ${ }^{7}$ and Munetaka Ueno ${ }^{5}$ \\ ${ }^{1}$ Loránd Eötvös University, Departement of Astronomy, Pázmány Péter sétány 1, 1117 \\ Budapest, Hungary, email: S.Zahorecz@astro.elte.hu \\ ${ }^{2}$ Konkoly Observatory of the Hungarian Ac. of Sci., PO Box 67, 1525 Budapest, Hungary \\ ${ }^{3}$ Department of Physical Science, Osaka Prefecture University, Gakuen 1-1, Sakai, Osaka \\ 599-8531, Japan \\ ${ }^{4}$ National Astronomical Observatory of Japan, 2-21-1 Osawa, Mitaka, Tokyo 181-8588, Japan \\ ${ }^{5}$ JAXA, 3-1-1 Yoshinodai, Sagamihara, Kanagawa, 229-8510, Japan \\ ${ }^{6}$ Research Institute for Soil Science and Agricultural Chemistry of the Hungarian Academy of \\ Sciences, GIS Lab, Budapest, Hungary \\ ${ }^{7}$ College of Arts and Sciences, UPLB College, Laguna 4031 Philippines
}

Abstract. Physical parameters were derived for 100 young stellar objects in the TAPO region.

Keywords. stars: formation — stars: pre-main-sequence — infrared: stars

We made an analysis of 4441 AKARI FIS (Kawada et al. 2007; Yamamura et al. 2010) point sources in the Tau-Aur-Per and Orion region. Spectral energy distribution (SED) was drawn for about 550 point sources based on AKARI FIS, AKARI IRC (Ishihara et al. 2010), Spitzer Space Telescope (SST, Werner et al. 2004) Infrared Array Camera (IRAC; Fazio et al. 2004), Mid-Infrared Photometer for Spitzer (MIPS; Rieke et al. 2004), 2 Micron All Sky Survey (Skrutskie et al. 2006) and Wide-Field Infrared Survey Explorer (WISE, Wright et al. 2010) and various other photometric data. We determined the physical parameters (e.g. stellar mass, temperature and radius, disk size and mass) of 100 YSOs with the SED Fitting Tool of Robitaille et al. 2007 and for other 450 sources we determined an evolutionary stage based on the slope of the SED in mid-IR and FIR.

Acknowledgement. The European Union and the European Social Fund have provided financial support to the project under the grant agreement no. TAMOP-4.2.1/B09/1/KMR-2010-0003. This research was partly supported by the Hungarian Research Fund (OTKA) and the HAS-JSPS mobility program.

\section{References}

Ishihara, D., Onaka, T., Kataza, H. et al. 2010, A\&A, 514, 1

Fazio, G. G., Hora, J. L., Allen, L. E. et al. 2004, ApJS, 154, 10

Rieke, G. H., Young, E. T., Engelbracht, C. W. et al. 2004, ApJS, 154, 25

Kawada, M., Baba, H., Barthel, P. D., Clements, D. et al. 2007, PASJ, 59, 389

Robitaille, T. P., Whitney, B. A., Indebetouw, R., \& Wood, K. 2007, ApJS, 169, 328

Skrutskie, M. F., Cutri, R. M., Stiening, R. et al. 2006, AJ, 131, 1163

Yamamura, I. et al. 2010, AKARI/FIS Bright Source Catalogue Version 1.0 Release Note

Werner, M. W., Roellig, T. L., Low, F. J. et al. 2004, ApJS, 154, 1

Wright, E. L., Eisenhardt, P. R. M., Mainzer, A. K. et al. 2010, AJ, 140, 1868 